\title{
Unveiling the gene regulatory landscape in diseases through the identification of DNase I-hypersensitive sites (Review)
}

\author{
YING CHEN and AILING CHEN \\ Central Laboratory, The Affiliated Wuxi Maternity and Child Health Care Hospital \\ of Nanjing Medical University, Wuxi, Jiangsu 214002, P.R. China
}

Received December 11, 2018; Accepted July 3, 2019

DOI: $10.3892 /$ br.2019.1233

\begin{abstract}
DNase I-hypersensitive sites (DHSs) serve key roles in the regulation of gene transcription as markers of cis-regulatory elements (CREs). Recent advances in next-generation sequencing have enabled the genome-wide location and annotation of DHSs in a variety of cells. Numerous studies have confirmed that DHSs are involved in several processes in cell fate decision and development. DHSs have also been indicated in cancer and inherited diseases as driver distal regulatory elements. Here, the definition of DHSs is reviewed, in addition to high-throughput methods of DHS identification. Furthermore, the function of DHSs in gene expression is probed. The roles of DHSs in disease occurrence are also reviewed and discussed. Concomitant advances in the identification of essential roles of DHSs will assist in disclosing the underlying molecular mechanisms, supplementing gene transcription and enlarging the molecular basis of DHS-related bioprocesses, phenotypes, distinct traits and diseases.
\end{abstract}

\section{Contents}

1. Introduction

2. High-throughput sequencing methods for DHS identification

3. Functions of DHSs

4. Available data of DHSs

5. DHSs and diseases

6. Discussion

Correspondence to: Dr Ying Chen, Central Laboratory, The Affiliated Wuxi Maternity and Child Health Care Hospital of Nanjing Medical University, 48 Huaishu Street, Wuxi, Jiangsu 214002, P.R. China

E-mail: yingchen8@163.com

Key words: cis-regulatory elements, DNase I-hypersensitive sites, DNase-sequencing, low-input DNase-sequencing, single-cell DNase-sequencing

\section{Introduction}

Definition of DNase I-hypersensitive sites (DHSs). DNase I is an endonuclease with little DNA-sequence specificity (1). In the early 1960s, DNase I was used to probe how the nucleosome was organized (2). Weintraub and Groudine (3) found that active chromatin was prioritized for decomposition by this enzyme. These specific regions are termed DHSs, which are distinct markers of active chromatin that co-position with various cis-regulatory elements (CREs), including the expression regulation sequences of enhancers and promoters, negative regulation sequence of insulators, silencers, and certain locus control regions. DHSs usually disperse around transcriptionally active genes and are accessible to regulatory proteins. Therefore, by contrast, certain regions within DHSs are resistant to degradation as they are protected by these gene regulatory proteins, including transcription factors.

Generation and erasure of DHSs. The underlying molecular mechanisms of gene regulation remain to be fully elucidated. The first step of gene regulation is that the cells adopt an 'open' structure in response to external stimulation at a group of specific sequences located in certain chromatin regions. These exposed sequences are bound by site-specific transcriptional regulatory elements, leading to chromatin structure remodeling marked by significant accessibility to the nucleases (4). DHSs in the open chromatin serve important roles in chromatin rearrangement $(5,6)$, and are stimulated by the different state of histone acetylation $(7,8)$ and the binding capacity of the chromatin remodeling multiprotein complex (9). DHSs act as binding anchors of activator proteins and mediating cofactors, and interact with the preinitiation complex at promoters (2,10-14), regulating gene expression.

DHSs can be eliminated by site-specific factors. It was reported that DHS at a CCCTC-binding factor (CTCF)-dependent silencer can be eliminated due to the eviction of CTCF and remodeling of a nucleosome caused by inducible non-coding RNA transcription in the chicken lysozyme (15). DHSs are dynamic and fine-tuned by different classes of remodeling enzymes. For example, TFE3 combines with ACF to stimulate the occurrence of an active DHS site in the IgH intronic enhancer, whereas PU.1 has been demonstrated to recruit Mi2 $\beta$ and subsequently erase this DHS (16). 
Characteristic features of DHSs. In mammalian cells, $>3 \%$ of the genome is found to be DNase I-hypersensitive (17). To date, 290,000,000 DHSs have been recognized. Each tissue/cell type is represented by multiple distinguished DHS profiling derived from different individuals. DHSs are considered to be the one of the most useful discriminative features between cell types (18) and have several distinctive characters.

First, DHSs are typically characterized by high sensitivity to DNase I, particularly when the related gene is actively transcribed. Regions with high transcriptional activity are reported to be even more sensitive than those with no transcriptional activity. That is, there exists two states of DHSs, open and closed, in which the accessibility features of chromatin is increased or decreased, respectively, associated with gene expression (19).

Second, DHSs are short sequences of $\sim 200$ base pairs with low methylation, and the majority are no more than several hundred base pairs long. These low-methylation regions are co-positioned at or close to the transcription starting sites $(20,21)$, which affect gene transcription according to the degree of methylation.

Third, DHSs are representative markers of regulatory DNA and overlap with multiple CREs, including promoters, enhancers and active transcription sites (22). In addition, DHSs have underpinned the identification of other CREs, including insulators, silencers and locus control regions (10).

Fourth, although each tissue/cell exhibits distinguished DHS signatures, there exists specific core regions in DHSs that can be identified by sequence-specific DNA-binding proteins. The core regions are conserved in different cell types across species and are enriched with binding sites of HMG14 and HMG17 proteins (23).

\section{High-throughput sequencing methods for DHS identification}

The techniques used for DHS identification do not vary substantially, all of which are novel techniques based on high-throughput sequencing. The differences are compared in Table I.

DNase-sequencing (DNase-seq). Decades ago, Southern blot hybridization was the major method used to identify DHSs by characterizing digested DNAs following the titration of DNase I (24). However, the low-throughput nature of this strategy profoundly restricted its further application. Improvements and the wide application of the massively parallel sequencing technique has allowed high-resolution genome-scale mapping of various DHSs, which lays a foundation for assembling comprehensive catalogs of regulatory sequences $(25,26)$. The first method used for identifying thousands of DHSs simultaneously was introduced by Crawford et al (27). Firstly, the nuclei are cleaved by DNase I, and the two ends are then digested blunt using T4 DNA polymerase. The genome is then cleaved by adding BamHI and $B g l$ II. The digested blunt or sticky fragments are ligated into the pBluescript $\mathrm{SK}(+)$ plasmid and sequenced. This method enriches the sequence within the genome that relates to active chromatin and identifies DHSs on a genome-wide scale. Two years later, these high-throughput strategies were renewed by attaching a biotinylated linker to the DNase-digested ends (Fig. 1). The linker tags are used to extract short joint DNA sequences, which can be identified by DNase-based high-throughput next-generation sequencing (DNase-seq) (28) or DNase-based microarrays (DNase-chip) (26). Similar strategies providing accurate mapping of DHSs further assist in revealing a large category of CREs in all types of mammalian tissues and cells $(29,30)$.

Morin et al (31) simulated the whole exon sequencing paradigm, and developed a customized capture panel for known DHSs ('immune sequences'), specific for DHS detection in immune cells and genetic variation in immune-related diseases.

Single-cell DNase-seq (scDNase-seq). Despite the robustness of DNase-seq technology, millions of cells are required, which limits its application in rare cases with limited cells, such as in certain cells from patients. In addition, traditional DNase-seq suffers from low sensitivity as a result of DNA loss during the multiple purification steps. Therefore, scDNase-seq, also known as Pico-seq, was developed to minimize DNA loss, and has been applied in the analysis of chromatin accessibility using single cells $(32,33)$. To prevent loss of the small quantity of DNase I-hypersensitive DNA released by DNase I digestion of single cells, a large amount of circular plasmid DNA is added as carrier DNA in the subsequent steps of library preparation (Fig. 2). The previous application of scDNase-seq to tumor cells, NIH3T3 cells and pools of normal cells has shown that DHS patterns at the single-cell level are highly reproducible among individual cells (33). This method enables the generation of a genome-wide DHS map for rare samples, which is more valuable for clinical application.

Low-input DNase-seq (liDNase-seq). Although scDNase-seq can identify DHSs from a small quantity of starting material, the annotation of sequencing results requires a pre-known DHS database, which is a challenge in the identification of de novo DHSs sites. Lu et al (34) introduced liDNase-seq by modifying the scDNase-seq method to achieve de novo genome-wide DHS identification using no more than 30 cells. The major technical improvements, including reducing the complexity of the purification process prior to the adaptor ligation reaction and the first amplification reaction, and modifying the size selection step by using SPRI affinity beads in place of gel purification. The process for generating DHS maps is similar to that of the ENCODE project (www.encodeproject.org and http://genome. ucsc.edu/ENCODE) and allows the identification of de novo DHSs at much higher resolution than in previous methods.

ImmunoSEQ technique. Morin et al (31) developed an ImmunoSEQ technique for the detection of known DHSs. Using this technique, whole-exome sequencing or customized DHS region sequencing can be efficiently performed. The analysis focuses on the variation of non-coding regions of immune-related diseases.

\section{Functions of DHSs}

DHSs are involved in gene expression regulation. DHSs are essential features of all defined types of active CREs and 
Table I. Comparison of different techniques to identify DHSs.

\begin{tabular}{llclclcc}
\hline Technique & \multicolumn{1}{c}{$\begin{array}{c}\text { Cell } \\
\text { requirement }\end{array}$} & $\begin{array}{c}\text { Data } \\
\text { interpretation }\end{array}$ & $\begin{array}{c}\text { Novel DHS } \\
\text { identification }\end{array}$ & $\begin{array}{c}\text { Customized } \\
\text { DHSs }\end{array}$ & $\begin{array}{c}\text { Complex } \\
\text { process }\end{array}$ & $\begin{array}{c}\text { DNase I } \\
\text { digestion }\end{array}$ & Resolution \\
\hline DNase-seq & Large number of cells & Difficult & Yes & No & Yes & Yes & High \\
scDNase-seq & One or $\sim 100$ cells & Difficult & No & No & Yes & Yes & Low \\
liDNase-seq & $<30$ cells & Difficult & Yes & No & Reduced & Yes & High \\
ImmunoSEQ & Large number of cells & Easy & Customized & Yes & Simpler & No & \\
& & & DHS region & & & & \\
& & & & & & &
\end{tabular}

Accurate DHSs, DNase I-hypersensitive sites; DNase-seq, DNase-sequencing; scDNase-seq, single-cell DNase-seq; liDNase-seq, low-input DNase-seq.

are often co-positioned with them (35-37). DHSs are directly involved in chromatin modeling and structural reestablishment, the recognition of regulatory proteins and regulating the initiation of transcription. DHSs are associated with nearby gene expression changes through the binding of certain regulatory proteins to their specific sequence at promoters or other CREs regions, and are thus involved in cell fate decisions, individual variation and development (22). Frank et al (19) detected thousands of CREs at which the accessibility of chromatin increased or decreased. These changes coincided with the transcription level of adjacent genes, which is important in the regulation of global gene expression, and most likely infers activation or deactivation of enhancer elements. Huang and Liew (38) identified DHSs in the 4-kb upstream locus of the cardiac myosin heavy chain- $\alpha$ (MHC- $\alpha$ ) gene in the hamster and revealed a conserved GATA-motif site that interacts specifically with GATA-binding factors at different stages of cardiomyocyte development, which provided evidence for the role of GATA factors in the gene expression of cardiac MHC- $\alpha$.

Open DHSs often mark increases in local transcription levels, which supports the observation that open DHSs are enhancers. Similarly, closed DHSs may represent reduced enhancer activity (19). This influence is more apparent when genes are associated with two or more directional matched DHS changes. The findings identified in genome-wide association studies (GWASs) show that genetic variations frequently lie in non-coding regions of the genome that contain CREs, which suggests that gene expression change underlies the development of several complex traits.

DHSs exhibit distinguished profiles and contribute to define CREs. Cells in a specific stage and stature possess a fixed set of CREs that are accessible to trans-acting factors, and thus underlie a complex controlling network of chromatin $(35,39)$. Each cell type has a specific set of regulatory sequences and the cumulative span of those sequence consists of $>80 \%$ of the non-coding region of the genome. Studies on DHSs help to disclose delicate gene regulation mechanisms and enable extensive annotation of the genome. The genome-wide mapping of DHSs provides a novel platform for the promising investigation of a specific molecular biological problems affected by the regulation of a given gene or a group of genes (17).
In addition, DHSs form a complicated, spatially- and temporally-specific network. Certain DHSs identified in one cell type by DNase-seq may not occur in the other cell types. Pan et al (40) reported that 12 DHSs in chromatin related to the Msx 2 gene varied in different cell types in the chicken, when they examined anterior and posterior limb mesenchymal cells, calvarial osteoblasts and fibroblasts in embryos. Most of the DHSs were not detected as active in any of the four typed of cells, and only the DHS in the basal promoter region was present in all four tissues. One DHS was active and unique in the cells with Msx 2 transcripts, and a secondary DHS was unique in non-expressing cells. The anterior and posterior limb mesenchyme cells had a distinct group of DHSs, which were more complex than those detected in calvarial osteoblast cells, which suggested that a complicated DHS pattern may be involved in the different regulation models of the Msx2 gene in these two tissues, and is involved in cell fate decision by interaction with cell-specific transcription factors to guide the transcription program of cell fate decision and development. DHSs of a certain gene may also change in response to different transcription activity. Grünweller et al (41) examined the 5'-end of the vigilin gene in chickens using the DNase-seq technique and reporter gene analysis method. They identified two candidate DHSs. One DHS was active and unique under high transcriptional activity of the vigilin gene promoter in the chicken cells, which was termed DHS1, and a secondary DHS was only found under low transcriptional activity, which was termed DHS2. The activity of the promoter of the vigilin gene was enhanced over 10 times by upstream sequences of the transcription start site (TSS). Identifying DHSs and comparing their features differs among various cell types or within a similar cell type, but culture in different circumstances is essential for revealing gene expression patterns under different conditions. This can effectively complement current understanding and may have potential clinical applications for disease treatment. The exploitation of variable and plastic patterns of active DHSs offers potential for the identification of certain cell or tissue states, which may have potential to be applied to clinical diagnoses and predictions or the evaluation of therapeutic effects.

Studies investigating DHSs facilitate the identification of novel CREs, as DHSs are more promising indicators for the identification of chromatin accessibility, which have been widely used to map functional regulation elements. DHSs 

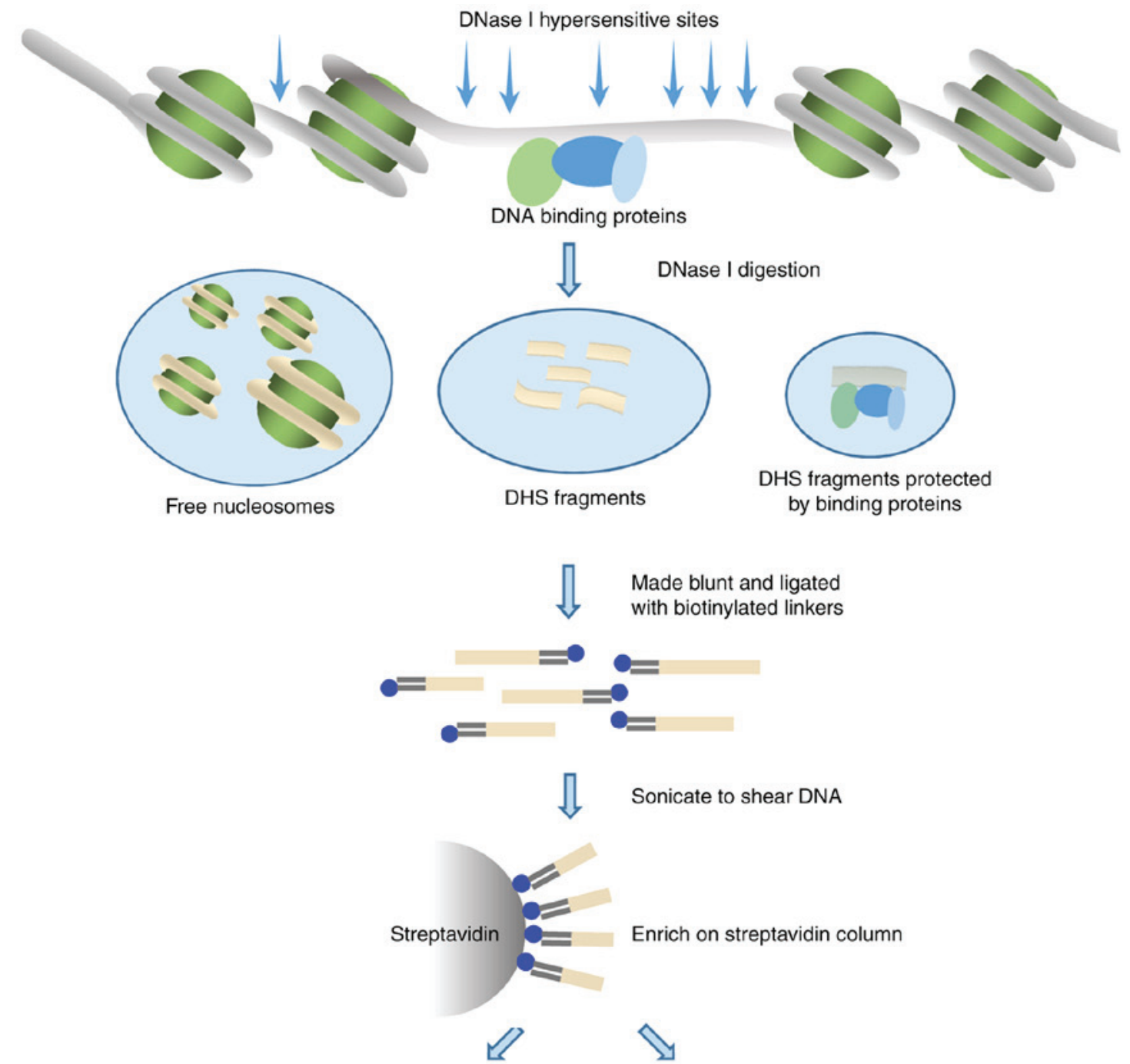

Enrich on streptavidin column

Hybridize to tiled arrays

Library construction

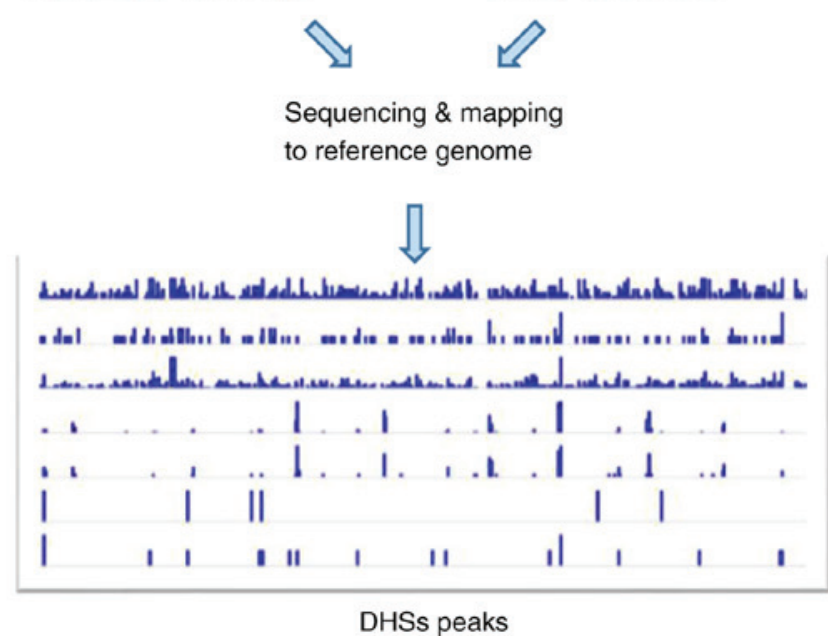

Figure 1. Experimental procedures of the high-throughput DHSs identification protocol. Intact nuclei are digested with DNase I and then made blunt, followed by ligation of a biotinylated linker and sonication for shearing. The products are incubated on a streptavidin column for pooling of the DNase I-cleaved ends. The extracted short adjacent DNA fragments are either hybridized to tiled arrays (DNase-chip) or subjected to library construction and next generation sequencing (DNase-sequencing). DHSs, DNase I-hypersensitive sites.

overlie CREs with parallel degrees of nuclease sensitivity and cover the main sequence of regulatory factor (42). DHSs usually contain CREs related to transcriptional activation on the reporter locus, such as enhancers, but can also contain transcription inhibition, such as silencers (17). A DHS map reveals the state and pattern of the presence of CREs, in addition to 

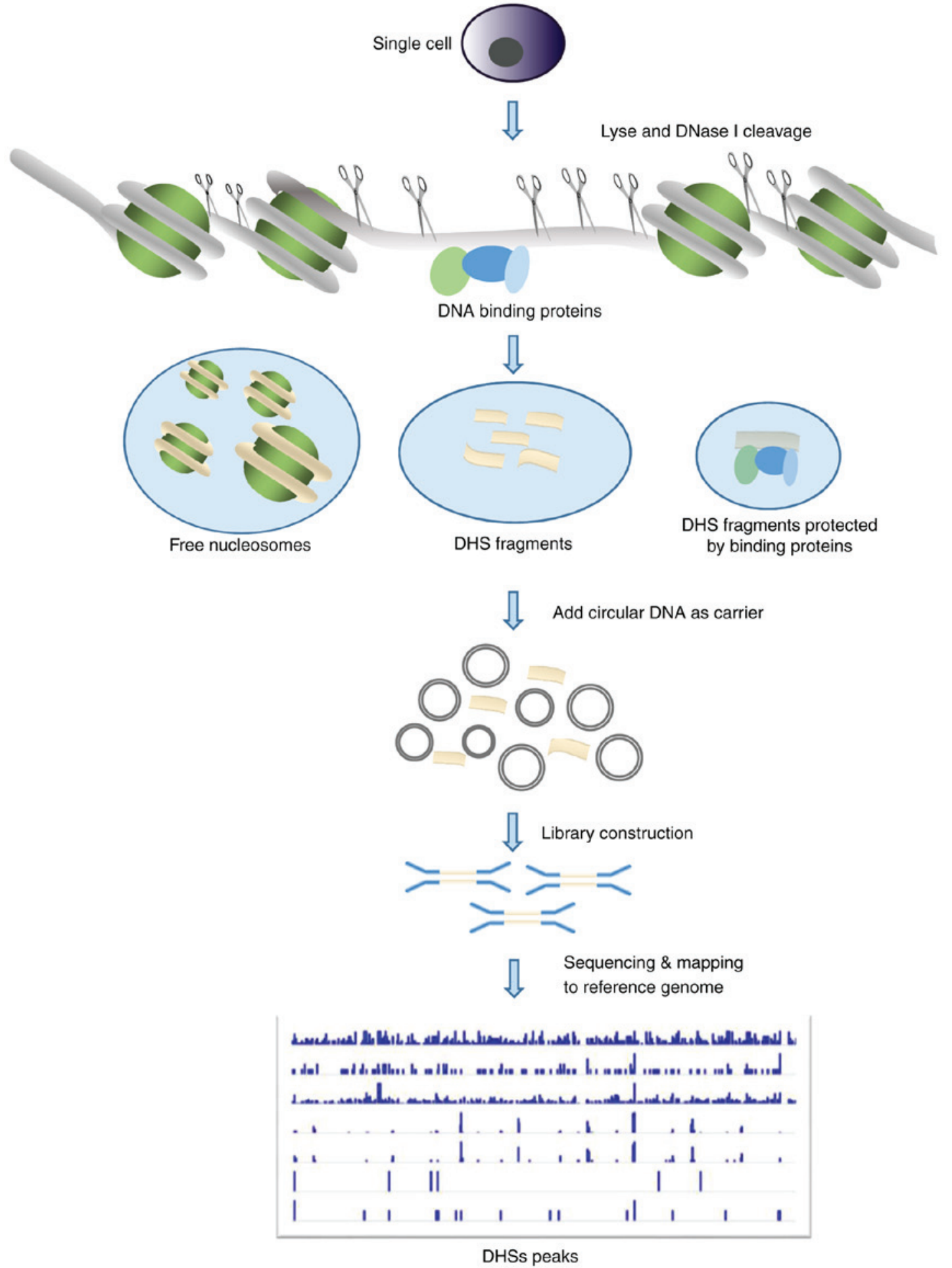

Figure 2. Schematic of single-cell DNase sequencing. Intact nuclei are digested by DNase I and followed by library construction including end-repair, ligation of the adaptors, PCR amplification with circular carrier DNA and high-throughput sequencing. DHSs, DNase I-hypersensitive sites.

the variable and plastic states of chromatin in various cell types $(25,29,33,43)$. Liu et al (44) identified 17,472 specific DHSs and transcription factor binding sites in two cell lines, the hESC H1 cell line and trophoblast (TB)-treated cell line, and constructed a transcription factor network for placental development. The specific DHSs in the TB-treated cells were found in the 'blood vessel' and 'trophectoderm', including members of the transcription factor motif family: Leucine zipper, helix-loop-helix, GATA and ETS. The model of a TB system induced by bone morphogenetic protein 4 (BMP4) was demonstrated to be important in investigating the mechanism of trophoblast development and revealed novel candidate genes involved in the regulation of human placental development. These findings indicate that DHSs enable the precise delineation of genomic CREs. Further investigations on DHSs are expected to reveal more novel regulatory elements.

Identifying sequence variations of the DHSs of phylogenetic trees instead of the coding region of genes may assist in 
disclosing the changes and evolution of certain phenotypes. Dong et al (45) analyzed and evaluated the accelerated evolution of orthologous sequences at DHSs from the human genome and primate genomes using systematic biology methods, and constructed a comparison map between the DHSs and ancient repeat elements (AREs). Their analysis identified the local AREs of all DHSs and demonstrated that they were neutrally evolving. Therefore, it is noteworthy that $\sim 0.44 \%$ of DHSs in the human genome are undergoing accelerated evolution (termed ace-DHSs). Further analysis of ace-DHSs is warranted for investigating the evolution of human-specific phenotypes. These DHS analyses are important in basic studies and may be of potential value in translational medicine and personalized medicine.

\section{Available data of DHSs}

The ENCODE project (www.encodeproject.org and http://genome.ucsc.edu/ENCODE) aims to evolve comprehensive schemes to list all human DHSs in order to map and catalog genome-wide CREs. DHSs mark transcriptionally active sites of chromatin, which may be the origin of cell selectivity.

The ENCODE research institutes have performed genome-wide mapping of DHSs in $>100$ human cell and tissue types, and almost 3,000,000 DHSs have been identified, including 71 normal differentiated primary cells, 16 immortalized primary cells, 30 malignancy-derived cell lines and eight multipotent and pluripotent progenitor cells. The 20-50-bp reads from the DNase-seq experiments enabled unique mapping to $86.9 \%$ of the genomic sequence, allowing the interrogation of a large fraction of transposon sequences. The DHS profiles of 125 different human cell types were obtained, and of these, $34 \%$ were specific to individual cell types and only a minority were detected in all cell types $(3,692)$. The open state of DHSs varied $>100$ times, but the constitution pattern was consistent in distinct cell types. It was demonstrated that $\sim 5 \%$ of DHSs were detected in the TSS region, while the remaining 95\% represented distal DHSs dispersed uniformly in intronic and intergenic regions. These data provide additional information for disclosing the mechanism of transcription.

\section{DHSs and diseases}

DHSs are associated with multiple diseases and have been suggested to serve distinct roles in the etiology of cancer, immune-related diseases, inflammatory bowel disease, Alzheimer's disease, bone marrow density problems, coronary artery disease, autism, and certain common diseases and complex traits (Fig. 3) (46). Recent evidence demonstrates the potential value of cell-specific and disease-related DHSs in personalized medicine. Evidence showing high overlap between human diseases and CREs has been well-documented, which confirm that 'critical' cell types may function as causal factors for certain diseases or help to maintain certain phenotypic traits (46).

The accessibility or inaccessibility of the state of DHSs is reported to be associated with diseases. An increasing number of novel DHSs have been found to be associated with diseases. Specific cell and tissue types have been identified as being associated with different diseases. For example, specific immune cell types are involved in immune-related diseases (inflammatory bowel disease), and specific tissue types are involved in diseases affecting specific organs (coronary artery disease), with other associations including adrenal glands in coronary artery disease, immune systems in Alzheimer's disease and kidneys with bone marrow density (46).

Thousands of tumor-specific DHSs located at promoter and enhancer regions have been detected, which have been shown to be involved in the occurrence and development of cancer.

Function-related mutations of the DHS region are closely correlated with transcription initiation activity and thus result in the occurrence of certain diseases (Fig. 4). There are $>100$ studies on DHSs that assessed various cell or tissue types by ENCODE Alliance (124 different cell types) and NIH roadmap epigenomics group (342 different adult fetal tissue samples), which demonstrate that an overlap exists between mutations at non-coding DNA regulatory sequences and diseases and traits.

GWASs have identified numerous single nucleotide polymorphisms (SNPs) at DHSs, which are associated with various types of quantitative traits and complex disorders. Local mutation density is variable throughout the genome (47). A study on 1,161 human cancer genomes revealed that the density of point mutations at the center of the DHS in the gene promoter region of somatic cells was increased (48). Numerous tissue types, including brain, pancreatic and liver tissue, have also demonstrated the enrichment of SNPs associated with DHSs in major depressive disorder (49). A 14-kb Down syndrome cell adhesion molecule deletion sequence, containing $12 \mathrm{CNS}$ DHSs, was found in an autistic family, with regulatory potential affecting the biology of the central nervous system (50). De novo mutations, rich in DHSs and proximal genes, have been significantly predicted to result in the loss of transcription binding factors. For example, deletion of lysine-specific demethylase $5 \mathrm{~B}$ binding was found at the promoter of the candidate autism risk gene, EFR3A (51).

A mutation at the SNP (chr18:52417839 G>C) site was reported to be correlated with follicular thyroid cancer, which had an influence on the binding of tumor suppressor protein p53 and subsequently resulted in the decreased gene expression of thioredoxin-like 1 (TXNL1) (33). The highest prevalence of mutations was found in the hypothetical driving factor DHS chr5:1325957-1328153, located in an intron of the Cleft lip and palate transmembrane 1-like (CLPTM1L) gene and $30 \mathrm{~kb}$ upstream of telomerase reverse transcriptase (TERT), results in the overexpression of six adjacent genes and four of these genes [TERT, CLPTM1L, thyroid hormone receptor interactor 13 (TRIP13), lysophosphatidylcholine acyltransferase 1 (LPCAT1)] are known to be associated with cancer (52-54).

Recently, a statistical method has been developed to identify distal regulatory elements with hypothetical driving mutations in breast cancer, to identify DHSs in non-coding genomic sequences associated with significant mutations in breast cancer and abnormal expression of adjacent genes, which may be important in the development of cancer (55). The mutation of chr5:1325957-1328153 at the DHS region in breast cancer was reported to be associated with the overexpression of oncogene tripartite motif containing 27 (TRIM27). In addition, abnormal activity was found with the mutation of chr6:28948439-28951450 at the DHS region. Using data from The Cancer Genome Atlas (TCGA) and breast cancer 


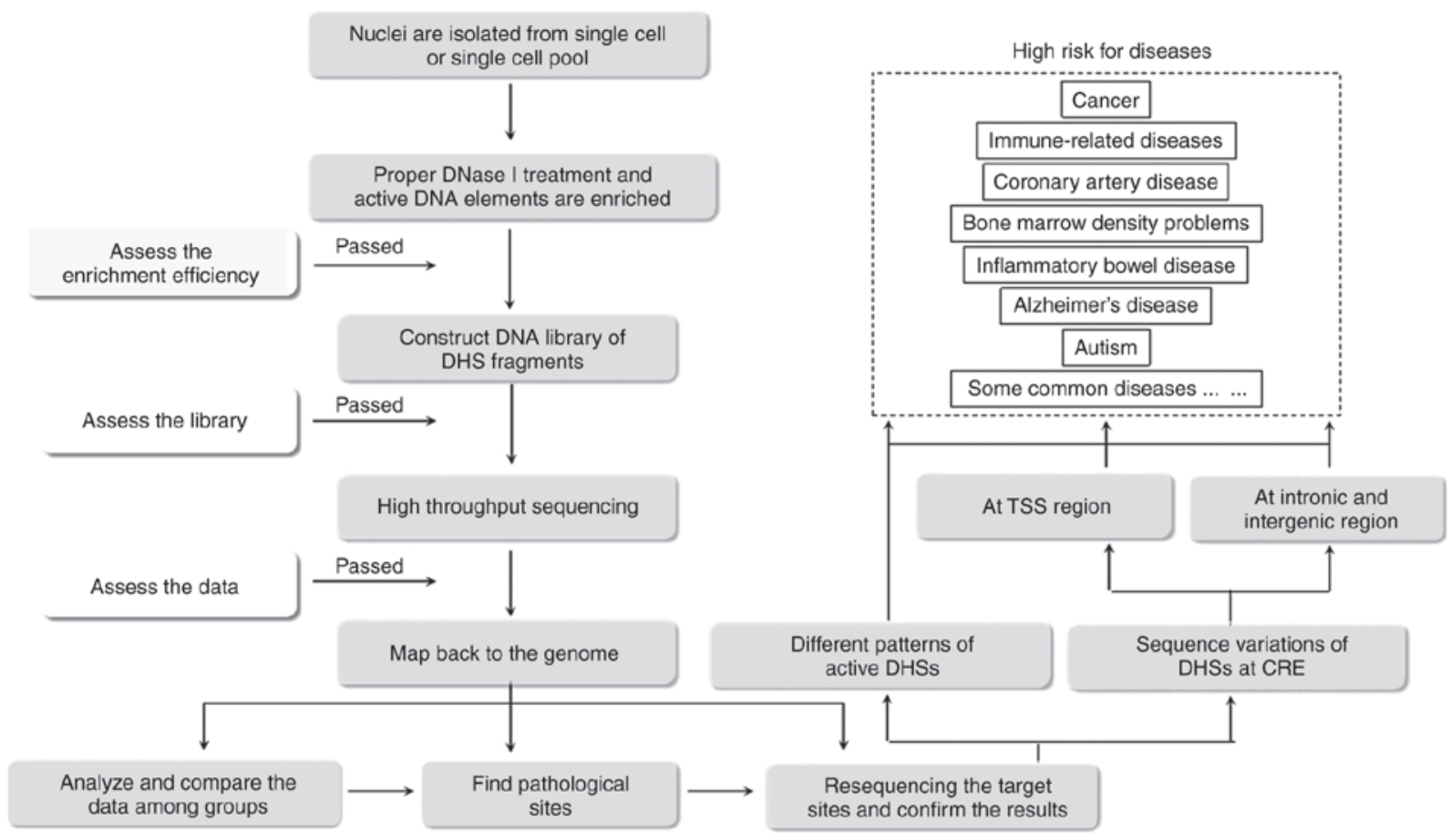

Figure 3. Process of identification of disease-related DHSs. DHSs, DNase I-hypersensitive sites; CRE, cis-regulatory element; TSS, transcription start site.

International Alliance (metabonomics) molecular taxonomy, Guo et al (56) found two hypothetical functional variants, rs62331150 and rs73838678, located at the DHS site and transcription factor binding region. Among them, rs62331150 was associated with the expression of tet methylcytosine dioxygenase 2 (TET2) in normal breast tissue and tumor tissue. Two new SNP (rs12309362 and rs9970827) were found to be significantly associated with reducing the risk of hepatocellular carcinoma (HCC) by measuring the mutations at the peak of DHSs in 1,538 patients with HCC and 1,465 normal controls (57).

A study on endometriosis, by re-sequencing $1.29 \mathrm{mb}$ of the 9p21 region, revealed that the mutation of rs17761446 at the DHS was associated with endometriosis, the protective $\mathrm{G}$ allele at this site had a strong interaction with the ANRIL promoter. Further chromatin immunoprecipitation analysis confirmed that the protective $G$ allele also had a preferential binding capacity with transcription factor 7-like 2, EP300 and may be involved in the development of endometriosis (58).

Studies on prostate cancer and breast cancer cells have revealed that different DHS patterns of androgen receptor (AR) and estrogen receptor 1 (ESR1) were of high predictive value for hormone receptor binding and may be involved in the development of these types of cancer. The quantitative measurement of DHS changes can predict the binding sites of perturbation-inducible transcription factors (59).

Following activation of the transcription of AR in LNCaP cells, 244 upregulated and 486 downregulated accessible DHS regions were detected to be the candidate sites for further investigation, which may be associated with prostate cancer. CTCF and the ELK1-ETS transcription factor are potential upstream regulator elements, which are rich in open promoter regions of downregulated genes. The inhibitor of DNA-binding $1 \mathrm{HLH}$ protein (ID1) is the only transcription factor that is significantly upregulated, exhibiting basal sequence enrichment in the promoter region of the upregulated gene. Therefore, CTCF, ELK1 and ID1 may be potential targets for the treatment of prostate cancer (60). Increasing evidence shows that changes in the expression of BMP4 are involved in the pathogenesis of cancer, which is associated with cancer metastasis and progression, including rectal, hepatocellular and ovarian cancer (61). In order to determine the characteristics of BMP4 transcription mediators in breast cancer, RNA-Seq and DNase-seq were analyzed in T-47D and MDA-MB-231 breast cancer cells treated with BMP4. It was confirmed that MBD2, core-binding factor- $\beta$ and hypoxia-inducible factor $1 \alpha$ were downstream regulators of the BMP4 signal, which enhanced cell migration and decreased cell growth (62).

In tumor therapy, particularly in acute myeloid leukemia (AML), intratumoral heterogeneity caused by clonal evolution has been found, which may have an influence on the effect of treatment. In order to solve this problem, the chromatin accessibility of subclones of AML was compared directly using unsupervised clustering analysis. Marked differences in the chromatin landscape and transcriptional regulation among the subclones were detected and confirmed. The data indicated that the common DHSs of individual AML subclones dominated in the clustering analysis over the subclone-specific DHSs, most likely due to the impact of shared founder mutations at the DHSs in each AML subclone. Clone-specific DHSs, runt-related transcription factor and ETS motifs are expressed in abundance in the two clones of DHSs, although GATA motifs are particularly abundant in FLT3-WT clones (63). It may be a potential strategy to use DHSs analysis to improve the treatment effect, particularly for those types of cancer with features of intraturmoral heterogeneity.

Genetic variation at DHSs has been reported to be correlated with carcinogenesis (64). By analyzing 1,161 human 
A

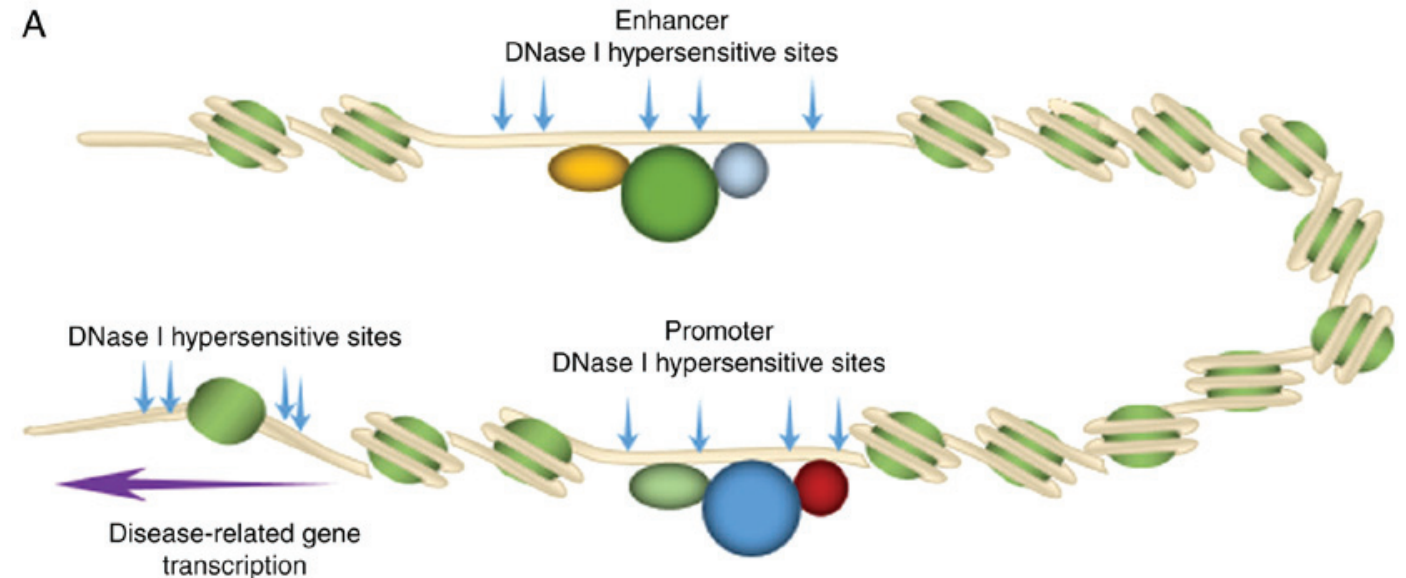

DNase I hypersensitive sites

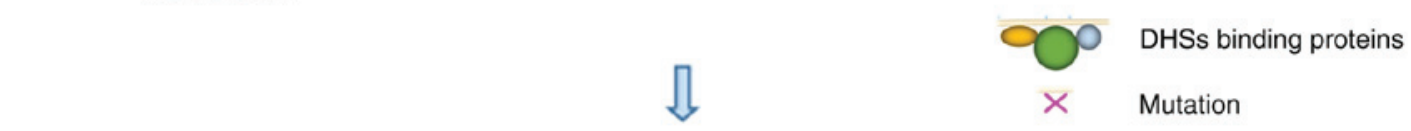

B

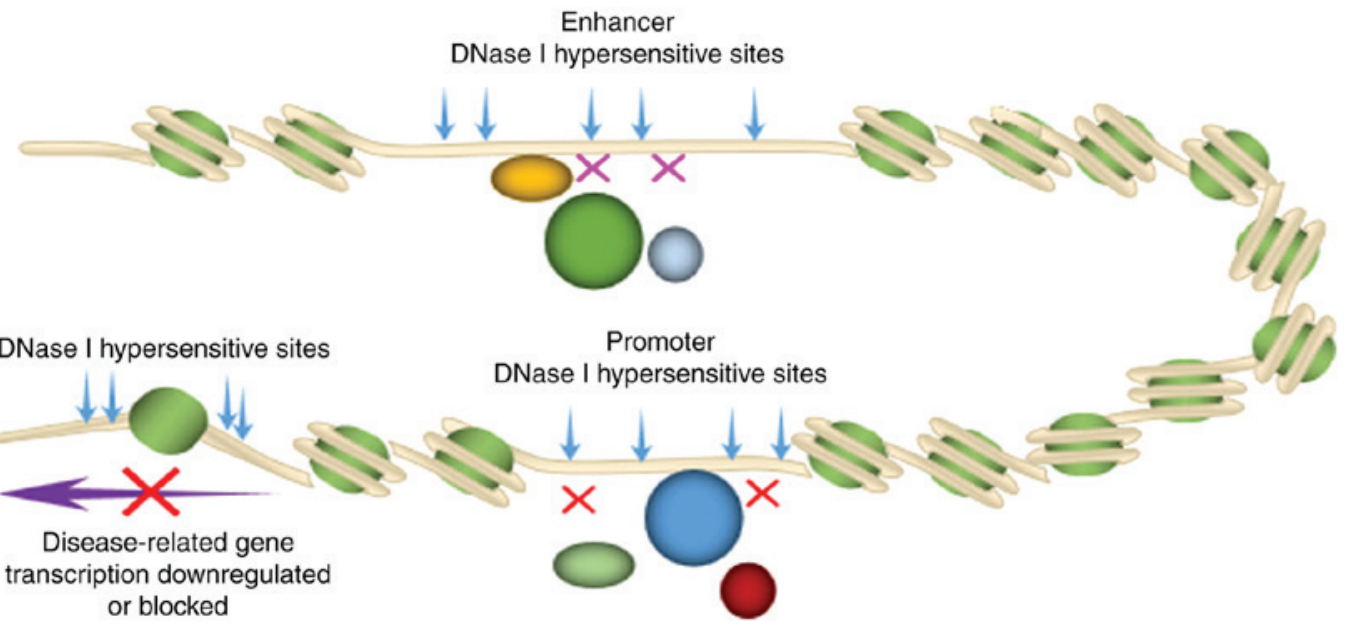

Figure 4. Pathological mechanism of variation at DHS sites of disease-related genes. (A) DHSs act as binding anchors of activator proteins and cofactors. A multiprotein complex is assembled and bound to the 'open' sequences of DHSs, including promoter or enhancer regions, and after chromatin rearrangement this leads to gene transcription. (B) When pathological mutations or variations occur at crucial regions of DHSs, it influences the identification and interaction of regulatory binding proteins and the transcription function is interrupted or altered, which may subsequently cause phenotypes. DHSs, DNase I-hypersensitive sites.

cancer samples from 14 types of cancer, DHS profiles and SNP distributions were mapped to link to promoter activity, some of which were involved in differential nucleotide excision repair (NER) and resulted in carcinogenesis (48). Consistent with this finding, genome-wide maps of NER regions show that the repair ability of nucleotide excision was decreased with mutation at the DHS of gene promoter regions.

Jin et al (33) reported that thousands of tumor-specific DHSs were identified on cells dissected from follicular thyroid carcinoma samples fixed on formalin-fixed paraffin-embedded slides. Numerous DHSs have been reported to be correlated with the development of thyroid cancer (33). A de novo mutation (chr18:52417839 G>C) at a DHS located downstream of the TXNL1 gene is associated with the formation of thyroid carcinoma. It was reported that rs62331150 located at promoter region and rs73838678 located at the enhancer region of the gene, increased the risk of breast cancer. These two SNP sites were found to be in linkage disequilibrium with rs9790517 of the adjacent TET 2 gene (55). It was also found that, in samples with mutation at the rs12309362 and rs9970827 sites, the risk of being affected with HCC decreased significantly (57).

Ten DHSs were identified as being mutated with abnormal expression of target genes in breast cancer (55). Mutation at the DHS chr5:1325957-1328153, was present at a high frequency in the cancer cells, resulting in the high level of transcription of certain genes close to it, including TERT, CLPTM1L, TRIP13 and LPCAT1, which has been confirmed to be associated with cancer $(52,53,65)$. Mutations at DHSs chr5:1325957-1328153 were found to result in the high expression of TRIM27, and certain mutations in this region caused abnormal accessibility of DHS chr6:28948439-28951450, which are associated with cancer.

The pattern of DHSs can be stimulated by hormones through regulation of the binding capacity of AR and ESR1 in prostate cancer cells and breast cancer cells. Following binding with AR or ESR1, the DNase I-hypersensitivity of certain sequences was found to be altered, and the regional nucleosome occupancy changed for AR binding but not for ESR1b binding, which indicated different interaction modes in AR and ESR1 regulation (59). 
In Gene Ontology analysis, genes associated with tumor-specific DHSs are abundant in biological processes, including the regulation of GTPase activity and response to hypoxia, and cancer-related pathways. Understanding the accessibility dynamics of chromatin in the process of disease occurrence and development can provide insights into how cell fate is regulated, and how transcriptional systems are organized and regulated in different tissues and how they are destroyed in disease states. In addition, the application of DHSs in biomedical research can expand the field of cell-selective gene regulation analysis, enabling the identification of long-range regulatory patterns of the system and previously undescribed phenomena, such as DHS activation patterns and mutation rates in abnormal and immortal cells.

\section{Discussion}

Although DHSs occupy a small portion of human genome, $\sim 2 \%$ of the genome, a relatively large proportion of CREs may be involved in the establishment of well-organized expression networks in each cell type and thus contribute to the etiology of a certain disease. The comprehensive delineation of distribution, constituents and biological activities of DHSs help to map and classify functional CREs. The identification of CREs is critical for elucidating the mechanism of gene expression regulation underlying biological events, and the development and progression of certain diseases.

To date, the DNase-seq technique remains one of the most efficient techniques for disclosing the known and unknown regulatory elements of diverse target cells. Cooper et al (32) released a detailed protocol in Nature that may facilitate the spread of DNase-seq analysis. However, the number of researchers able to utilize the high-throughput DHS capture technique well is limited. In addition, careful manipulation is required due to high background noise. Difficulties in performing experiments are not the greatest challenge for its wider application; bioinformatics analysis is difficult for the majority of laboratories. An insufficient number of bioinformatics technicians, particularly those with the required programming skills and familiarity in this field, is the main concern. The exploitation of software or online tools is required to simplify bioinformatics analysis and enable easier understanding by researchers.

Although evidence has identified an association between DHSs and diseases, using DHSs as a marker for prediction, prevention and pre-clinical diagnosis remains a challenge due to the complexity of regulation of the DHS profile. The uncertainty of genome-wide prediction of CREs and specific DHS related to gene function may increase the challenge of its application in clinical practice. Future work should focus on the investigation of more delicate methods to locate DHSs precisely, help disclose the mechanisms underlying gene expression differences, determine how to modify chromatin accessibility, reveal how changes in transcription factor binding are driven by genetic variations, and guide how to integrate DHSs in clinical practice.

\section{Acknowledgements}

Not applicable.

\section{Funding}

This study was supported by the National Natural Science Foundation of China (grant no. 81671473), the Key Talents of Jiangsu Province (grant nos. WSW-108 and FRC201754) and the Innovation Team Project of Wuxi (grant no. CXTDJS003).

\section{Availability of data and materials}

Not applicable.

\section{Authors' contributions}

YC conceived and wrote the manuscript with some assistance from AC.

\section{Ethics approval and consent to participate}

Not applicable.

\section{Patient consent for publication}

Not applicable.

\section{Competing interests}

The authors declare that they have no competing interests.

\section{References}

1. Elgin SC: DNAase I-hypersensitive sites of chromatin. Cell 27: 413-415, 1981.

2. Meisterernst M, Roy AL, Lieu HM and Roeder RG: Activation of class II gene transcription by regulatory factors is potentiated by a novel activity. Cell 66: 981-993, 1991.

3. Weintraub $\mathrm{H}$ and Groudine M: Chromosomal subunits in active genes have an altered conformation. Science 193: 848-856, 1976.

4. Felsenfeld G, Boyes J, Chung J, Clark D and Studitsky V: Chromatin structure and gene expression. Proc Natl Acad Sci USA 93: 9384-9388, 1996.

5. Wu C: The 5' ends of Drosophila heat shock genes in chromatin are hypersensitive to DNase I. Nature 286: 854-860, 1980.

6. Martinez-Balbas MA, Dey A, Rabindran SK, Ozato K and Wu C: Displacement of sequence-specific transcription factors from mitotic chromatin. Cell 83: 29-38, 1995.

7. Hebbes TR, Clayton AL, Thorne AW and Crane-Robinson C: Core histone hyperacetylation co-maps with generalized DNase I sensitivity in the chicken beta-globin chromosomal domain. EMBO J 13: 1823-1830, 1994.

8. Kleff S, Andrulis ED, Anderson CW and Sternglanz R: Identification of a gene encoding a yeast histone $\mathrm{H} 4$ acetyltransferase. J Biol Chem 270: 24674-24677, 1995.

9. Peterson CL and Tamkun JW: The SWI-SNF complex: A chromatin remodeling machine? Trends Biochem Sci 20: 143-146, 1995.

10. Roeder RG: The role of general initiation factors in transcription by RNA polymerase II. Trends Biochem Sci 21: 327-335, 1996.

11. Kim YJ, Bjorklund S, Li Y, Sayre MH and Kornberg RD: A multiprotein mediator of transcriptional activation and its interaction with the C-terminal repeat domain of RNA polymerase II. Cell 77: 599-608, 1994.

12. Koleske AJ and Young RA: An RNA polymerase II holoenzyme responsive to activators. Nature 368: 466-469, 1994.

13. Dynlacht BD, Hoey T and Tjian R: Isolation of coactivators associated with the TATA-binding protein that mediate transcriptional activation. Cell 66: 563-576, 1991.

14. Verrijzer CP and Tjian R: TAFs mediate transcriptional activation and promoter selectivity. Trends Biochem Sci 21: 338-342, 1996. 
15. Lefevre P, Witham J, Lacroix CE, Cockerill PN and Bonifer C: The LPS-induced transcriptional upregulation of the chicken lysozyme locus involves CTCF eviction and noncoding RNA transcription. Mol Cell 32: 129-139, 2008.

16. Ishii H, Du H, Zhang Z, Henderson A, Sen R and Pazin MJ: Mi2beta shows chromatin enzyme specificity by erasing a DNase I-hypersensitive site established by ACF. J Biol Chem 284: 7533-7541, 2009.

17. Zeng WP and McFarland MM: Rapid and unambiguous detection of DNase I hypersensitive site in rare population of cells. PLoS One 9: e85740, 2014.

18. Trynka G, Sandor C, Han B, Xu H, Stranger BE, Liu XS and Raychaudhuri S: Chromatin marks identify critical cell types for fine mapping complex trait variants. Nat Genet 45: 124-130, 2013.

19. Frank CL, Manandhar D, Gordan R and Crawford GE: HDAC inhibitors cause site-specific chromatin remodeling at PU.1-bound enhancers in K562 cells. Epigenetics Chromatin 9: 15, 2016.

20. Choi YC and Chae CB: DNA hypomethylation and germ cell-specific expression of testis-specific $\mathrm{H} 2 \mathrm{~B}$ histone gene. J Biol Chem 266: 20504-20511, 1991.

21. Ngo V, Gourdji D and Laverriere JN: Site-specific methylation of the rat prolactin and growth hormone promoters correlates with gene expression. Mol Cell Biol 16: 3245-3254, 1996

22. Zhang T, Marand AP and Jiang J: PlantDHS: A database for DNase I hypersensitive sites in plants. Nucleic Acids Res 44: D1148-D1153, 2016.

23. Deng T, Zhu ZI, Zhang S, Postnikov Y, Huang D, Horsch M, Furusawa T, Beckers J, Rozman J, Klingenspor M, et al: Functional compensation among HMGN variants modulates the DNase I hypersensitive sites at enhancers. Genome Res 25: 1295-1308, 2015.

24. Kodama Y, Nagaya S, Shinmyo A and Kato K: Mapping and characterization of DNase I hypersensitive sites in Arabidopsis chromatin. Plant Cell Physiol 48: 459-470, 2007.

25. Boyle AP, Davis S, Shulha HP, Meltzer P, Margulies EH, Weng Z, Furey TS and Crawford GE: High-resolution mapping and characterization of open chromatin across the genome. Cell 132: 311-322, 2008.

26. Crawford GE, Davis S, Scacheri PC, Renaud G, Halawi MJ, Erdos MR, Green R, Meltzer PS, Wolfsberg TG and Collins FS: DNase-chip: A high-resolution method to identify DNase I hypersensitive sites using tiled microarrays. Nat Methods 3: 503-509, 2006.

27. Crawford GE, Holt IE, Mullikin JC, Tai D, Blakesley R, Bouffard G, Young A, Masiello C, Green ED, Wolfsberg TG, et al: Identifying gene regulatory elements by genome-wide recovery of DNase hypersensitive sites. Proc Natl Acad Sci USA 101: 992-997, 2004.

28. Crawford GE, Holt IE, Whittle J, Webb BD, Tai D, Davis S, Margulies EH, Chen Y, Bernat JA, Ginsburg D, et al: Genome-wide mapping of DNase hypersensitive sites using massively parallel signature sequencing (MPSS). Genome Res 16: 123-131, 2006.

29. Thurman RE, Rynes E, Humbert R, Vierstra J, Maurano MT, Haugen E, Sheffield NC, Stergachis AB, Wang H, Vernot B, et al: The accessible chromatin landscape of the human genome. Nature 489: 75-82, 2012.

30. Vierstra J, Rynes E, Sandstrom R, Zhang M, Canfield T, Hansen RS, Stehling-Sun S, Sabo PJ, Byron R, Humbert R, et al Mouse regulatory DNA landscapes reveal global principles of cis-regulatory evolution. Science 346: 1007-1012, 2014.

31. Morin A, Kwan T, Ge B, Letourneau L, Ban M, Tandre K, Caron M, Sandling JK, Carlsson J, Bourque G, et al: Immunoseq: The identification of functionally relevant variants through targeted capture and sequencing of active regulatory regions in human immune cells. BMC Med Genomics 9: 59, 2016

32. Cooper J, Ding Y, Song J and Zhao K: Genome-wide mapping of DNase I hypersensitive sites in rare cell populations using single-cell DNase sequencing. Nat Protoc 12: 2342-2354, 2017.

33. Jin W, Tang Q, Wan M, Cui K, Zhang Y, Ren G, Ni B, Sklar J, Przytycka TM, Childs R, et al: Genome-wide detection of DNase I hypersensitive sites in single cells and FFPE tissue samples. Nature 528: 142-146, 2015.

34. Lu F, Liu Y, Inoue A, Suzuki T, Zhao K and Zhang Y: Establishing chromatin regulatory landscape during mouse preimplantation development. Cell 165: 1375-1388, 2016.

35. Gross DS and Garrard WT: Nuclease hypersensitive sites in chromatin. Annu Rev Biochem 57: 159-197, 1988.
36. Gaszner M and Felsenfeld G: Insulators: Exploiting transcriptional and epigenetic mechanisms. Nat Rev Genet 7: 703-713, 2006.

37. Li Q, Harju S and Peterson KR: Locus control regions: Coming of age at a decade plus. Trends Genet 15: 403-408, 1999.

38. Huang WY and Liew CC: A conserved GATA motif in a tissue-specific DNase I hypersensitive site of the cardiac alpha-myosin heavy chain gene. Biochem J 325: 47-51, 1997.

39. Bell O, Tiwari VK, Thoma NH and Schubeler D: Determinants and dynamics of genome accessibility. Nat Rev Genet 12: 554-564, 2011

40. Pan Z, Lichtler AC and Upholt WB: DNase I hypersensitive sites in the chromatin of the chicken Msx2 gene differ in anterior and posterior limb mesenchyme, calvarial osteoblasts and embryonic fibroblasts. Biochem Mol Biol Int 46: 549-557, 1998.

41. Grünweller A, Purschke WG, Kügler S, Kruse C and Müller PK: Chicken vigilin gene: A distinctive pattern of hypersensitive sites is characteristic for its transcriptional activity. Biochem J 326: 601-607, 1997.

42. Heintzman ND, Stuart RK, Hon G, Fu Y, Ching CW, Hawkins RD, Barrera LO, Van Calcar S, Qu C, Ching KA, et al: Distinct and predictive chromatin signatures of transcriptional promoters and enhancers in the human genome. Nat Genet 39: 311-318, 2007.

43. Sheffield NC, Thurman RE, Song L, Safi A, Stamatoyannopoulos JA, Lenhard B, Crawford GE and Furey TS: Patterns of regulatory activity across diverse human cell types predict tissue identity, transcription factor binding, and long-range interactions. Genome Res 23: 777-788, 2013.

44. Liu Y, Ding D, Liu H and Sun X: The accessible chromatin landscape during conversion of human embryonic stem cells to trophoblast by bone morphogenetic protein 4. Biol Reprod 96: 1267-1278, 2017.

45. Dong X, Wang X, Zhang F and Tian W: Genome-wide identification of regulatory sequences undergoing accelerated evolution in the human genome. Mol Biol Evol 33: 2565-2575, 2016.

46. Mokry M, Harakalova M, Asselbergs FW, de Bakker PI and Nieuwenhuis EE: Extensive association of common disease variants with regulatory sequence. PLoS One 11: e0165893, 2016.

47. D'Antonio M and Ciccarelli FD: Integrated analysis of recurrent properties of cancer genes to identify novel drivers. Genome Biol 14: R52, 2013

48. Perera D, Poulos RC, Shah A, Beck D, Pimanda JE and Wong JW: Differential DNA repair underlies mutation hotspots at active promoters in cancer genomes. Nature 532: 259-263, 2016.

49. Peterson RE, Cai N, Bigdeli TB, Li Y, Reimers M, Nikulova A, Webb BT, Bacanu SA, Riley BP, Flint J and Kendler KS: The genetic architecture of major depressive disorder in han chinese women. JAMA Psychiatry 74: 162-168, 2017.

50. Turner TN, Hormozdiari F, Duyzend MH, McClymont SA, Hook PW, Iossifov I, Raja A, Baker C, Hoekzema K, Stessman HA, et al: Genome sequencing of autism-affected families reveals disruption of putative noncoding regulatory DNA. Am J Hum Genet 98: 58-74, 2016.

51. Yuen RK, Merico D, Cao H, Pellecchia G, Alipanahi B, Thiruvahindrapuram B, Tong X, Sun Y, Cao D, Zhang T, et al: Genome-wide characteristics of de novo mutations in autism. NPJ Genom Med 1: 160271-1602710, 2016.

52. Wang K, Sturt-Gillespie B, Hittle JC, Macdonald D, Chan GK, Yen TJ and Liu ST: Thyroid hormone receptor interacting protein 13 (TRIP13) AAA-ATPase is a novel mitotic checkpoint-silencing protein. J Biol Chem 289: 23928-23937, 2014.

53. Rafnar T, Sulem P, Stacey SN, Geller F, Gudmundsson J, Sigurdsson A, Jakobsdottir M, Helgadottir H, Thorlacius S, Aben KK, et al: Sequence variants at the TERT-CLPTM1L locus associate with many cancer types. Nat Genet 41: 221-227, 2009.

54. Abdelzaher E and MostafaM F: Lysophosphatidylcholine acyltransferase 1 (LPCAT1) upregulation in breast carcinoma contributes to tumor progression and predicts early tumor recurrence. Tumour Biol 36: 5473-5483, 2015.

55. D Antonio M, Weghorn D, D Antonio-Chronowska A, Coulet F, Olson KM, DeBoever C, Drees F, Arias A, Alakus H, Richardson AL, et al: Identifying DNase I hypersensitive sites as driver distal regulatory elements in breast cancer. Nat Commun 8: 436, 2017.

56. Guo X, Long J, Zeng C, Michailidou K, Ghoussaini M, Bolla MK, Wang Q, Milne RL, Shu XO, Cai Q, et al: Fine-scale mapping of the 4q24 locus identifies two independent loci associated with breast cancer risk. Cancer Epidemiol Biomarkers Prev 24: 1680-1691, 2015. 
57. Jiang T, Du F, Qin N, Lu Q, Dai J, Shen H and Hu Z: Systematical analyses of variants in DNase I hypersensitive sites to identify hepatocellular carcinoma susceptibility loci in a Chinese population. J Gastroenterol Hepatol 32: 1887-1894, 2017.

58. Nakaoka H, Gurumurthy A, Hayano T, Ahmadloo S, Omer WH, Yoshihara K, Yamamoto A, Kurose K, Enomoto T, Akira S, et al: Allelic imbalance in regulation of ANRIL through chromatin interaction at 9p21 endometriosis risk locus. PLoS Genet 12: e1005893, 2016.

59. He HH, Meyer CA, Chen MW, Jordan VC, Brown M and Liu XS: Differential DNase I hypersensitivity reveals factor-dependent chromatin dynamics. Genome Res 22: 1015-1025, 2012.

60. Wei X, Yu L, Jin X, Song L, Lv Y and Han Y: Identification of open chromosomal regions and key genes in prostate cancer via integrated analysis of DNase-seq and RNA-seq data. Mol Med Rep 18: 2245-2252, 2018

61. Kallioniemi A: Bone morphogenetic protein 4-a fascinating regulator of cancer cell behavior. Cancer Genet 205: 267-277, 2012.

62. Ampuja M, Rantapero T, Rodriguez-Martinez A, Palmroth M, Alarmo EL, Nykter $M$ and Kallioniemi A: Integrated RNA-sec and DNase-seq analyses identify phenotype-specific BMP4 signaling in breast cancer. BMC Genomics 18: 68, 2017.
63. de Boer B, Prick J, Pruis MG, Keane P, Imperato MR, Jaques J, Brouwers-Vos AZ, Hogeling SM, Woolthuis CM, Nijk MT, et al: Prospective isolation and characterization of genetically and functionally distinct AML subclones. Cancer Cell 34: 674-689, 2018.

64. Stergachis AB, Neph S, Reynolds A, Humbert R, Miller B, Paige SL, Vernot B, Cheng JB, Thurman RE, Sandstrom R, et al: Developmental fate and cellular maturity encoded in human regulatory DNA landscapes. Cell 154: 888-903, 2013.

65. Wei C, Dong X, Lu H, Tong F, Chen L, Zhang R, Dong J, Hu Y, Wu G and Dong X: LPCAT1 promotes brain metastasis of lung adenocarcinoma by up-regulating PI3K/AKT/MYC pathway. J Exp Clin Cancer Res 38: 95, 2019.

This work is licensed under a Creative Commons Attribution-NonCommercial-NoDerivatives 4.0 International (CC BY-NC-ND 4.0) License. 